\title{
Experiências Simultâneas de Espalhamento de Raios X e Calorimetria Diferencial de Varredura (SAXS/WAXD/DSC) com Resolução Temporal Utilizando Radiação Síncrotron
}

\author{
Tomás S. Plivelic \\ Laboratório Nacional de Luz Síncrotron, SP \\ Silvana N. Cassu \\ Laboratório Nacional de Luz Síncrotron, SP \\ Centro Técnico Aeroespacial, SP
}

Maria do Carmo Gonçalves

Instituto de Química, UNICAMP

Iris L. Torriani

Laboratório Nacional de Luz Síncrotron, SP Instituto de Física "Gleb Wataghin", UNICAMP

\begin{abstract}
Resumo: Neste trabalho apresentamos uma nova instrumentação instalada na linha de SAXS do LNLS. Este equipamento permite a realização de experimentos simultâneos e resolvidos no tempo de espalhamento de raios $\mathrm{X}$ a baixos e altos ângulos (SAXS/WAXD) e calorimetria diferencial de varredura (DSC). O dispositivo de DSC mostrou-se comparável a equipamentos convencionais, com taxas de variação de temperatura de até $60^{\circ} \mathrm{C} / \mathrm{min}$ e uma precisão de $0.1{ }^{\circ} \mathrm{C}$. O uso de uma fonte de radiação síncrotron e de detetores de raios X sensíveis à posição permitiu a obtenção de dados com uma resolução temporal de $30 \mathrm{~s}$. A aplicação deste arranjo experimental no estudo da cristalização isotérmica e da fusão em materiais poliméricos é mostrada para o caso da policaprolactona (PCL) e suas blendas com polietileno clorado (PCL/ $\mathrm{PECl})$. As experiências mostraram a formação simultânea da estrutura cristalina e da morfologia lamelar nos diferentes estágios da cristalização assim como mudanças na cinética do processo com o tratamento isotérmico e a composição da blenda. Finalmente cabe destacar que experimentos simultâneos de SAXS/WAXD/DSC permitem o estudo de distintos processos abrangendo não apenas os de cristalização, mas também a formação de colóides e géis ou as transições de fase estruturais em diversos materiais.
\end{abstract}

Palavras Chave: SAXS, DSC, cristalização isotérmica, síncrotron.

\section{Simultaneous and Time Resolved X-Ray Scattering and Differential Scanning Calorimetry Experiments (SAXS/WAXD/DSC) using Synchrotron Radiation.}

Abstract: New instrumentation designed to perform simultaneous time-resolved X-ray scattering experiments at small and wide angles (SAXS/WAXD) as well as differential scanning calorimetry (DSC) has recently been installed at the SAXS beamline of the Laboratório Nacional de Luz Síncrotron. The DSC device proved to be comparable with conventional equipment, allowing temperature variation with rates of up to $60^{\circ} \mathrm{C} / \mathrm{min}$ with precision of $0.1^{\circ} \mathrm{C}$. The use of a synchrotron radiation source and position sensitive $\mathrm{X}$-ray detectors allows data collection in real time with $30 \mathrm{~s}$ resolution. The application of this experimental set-up in the isothermal crystallization and fusion of polymeric materials is given as an example. We present results of experiments with polycaprolactone (PCL) and its blends with chlorinated polyethylene (PCL/PECl), in which the simultaneous appearance of a crystalline structure and lamellar formation can be observed and the rate of process change for different compositions and thermal treatments can be determined. As a concluding remark, we mention that the SAXS/WAXD/DSC simultaneous experiments can also be performed with great advantage in the study of colloids and gel formation, as well as phase transitions in a variety of samples.

Keywords: SAXS, DSC, isothermal crystallization, synchrotron. 


\section{Introdução}

Experimentos simultâneos em função da temperatura são muito atrativos não só pela redução de erros devidos a diferenças no ambiente da amostra ou na história térmica do material, como também pela possibilidade de se determinar a seqüência de eventos de forma precisa e unívoca ${ }^{[1,2]}$. No caso particular de sistemas poliméricos, os experimentos simultâneos de espalhamento de raios $\mathrm{X}$ a baixos ângulos (SAXS) e difração de raios X (WAXD) combinados com calorimetria diferencial de varredura (DSC), permitem o acompanhamento das mudanças morfológicas e estruturais em escala nanométrica induzidas por tratamento térmico, podendo ser correlacionadas às transições de fase do sistema. A utilização de alto fluxo de fótons provenientes de radiação síncrotron, e de sistemas rápidos de deteção de raios $\mathrm{X}$ permite não somente uma estatística significativa das curvas de espalhamento, possibilitando o estudo in situ das transformações, como também o acompanhamento de fenômenos que acontecem em curtos períodos de tempo, tais como mecanismos de separação de fases e nucleação primária ${ }^{[3]}$, múltipla fusão e recristalização ${ }^{[4]}$. Assim, uma importante variedade de parâmetros estáticos e cinéticos de um sistema polimérico pode ser determinada contribuindo tanto na compreensão de aspectos fundamentais da físico-química de polímeros como na área de processamento desses materiais.

No presente trabalho será apresentada uma breve descrição do arranjo experimental de SAXS/WAXD/DSC que foi implementado na linha de SAXS do Laboratório Nacional de Luz Síncrotron (LNLS). A célula de DSC é uma adaptação de um estágio a quente utilizado em microscopia óptica que apresenta algumas particularidades para possibilitar experimentos simultâneos de calorimetria e de espalhamento de raios X. São mostrados resultados de sua resposta e uma comparação com sistemas convencionais de DSC. A potencialidade da ferramenta aqui descrita é aplicada no estudo da blenda binária miscível policaprolactona/ polietileno clorado, PCL/ PECl, onde o primeiro componente é cristalizável, enquanto que o segundo é amorfo. A obtenção de alguns parâmetros morfológicos e cinéticos da blenda é apresentada a partir do estudo da cristalização isotérmica e da fusão da PCL em função da composição da blenda e da temperatura de cristalização.

\section{Descrição do equipamento}

O calorímetro (DSC/THM 600, Linkam Ltd.) foi adaptado e instalado no banco ótico da linha de SAXS do LNLS ${ }^{[5]}$. Uma foto do equipamento em condições de trabalho é apresentada na Figura 1a.

A célula do DSC é composta por um forno de prata com uma fenda horizontal em sua parte central, a qual permite a transmissão dos raios $\mathrm{X}$ através da amostra. Esta célula opera no modo fluxo de calor e só uma amostra é tratada termicamente. O sinal de referência (porta amostra vazio) é descontado posteriormente. O forno do DSC opera entre -196 e $600{ }^{\circ} \mathrm{C}$ com taxas compreendidas entre os 0,1 a $60^{\circ} \mathrm{C} / \mathrm{min}$. O porta-amostra é uma

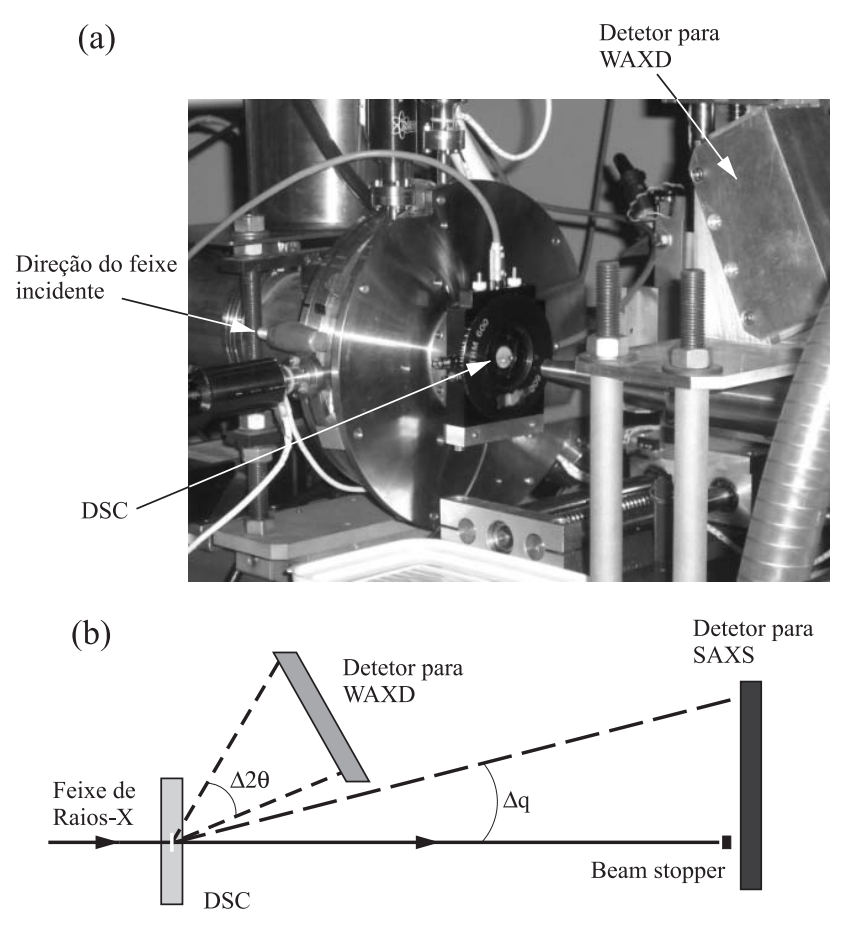

Figura 1. (a) Fotografia do arranjo experimental montado na linha de SAXS do LNLS. (b) Esquema do sistema utilizado nas experiências simultâneas de SAXS/WAXD/DSC.

cela convencional de alumínio para medidas de DSC, na qual foram feitos orifícios na sua parte central. Os orifícios são selados com janelas de mica de $30 \mu \mathrm{m}$ de espessura. A amostra a ser estudada é prensada entre as janelas de mica. Um esquema do arranjo completo utilizado é apresentado na Figura 1b. Os raios $\mathrm{X}$ provenientes de fonte de luz síncrotron e espalhados pela amostra são registrados em dois detetores a gás unidimensionais sensíveis à posição (Biologic Inc). A faixa angular de deteção para os experimentos aqui reportados foi de $0,008 \leq q \leq 0,21 \AA^{-1}$ para SAXS e $15 \leq 2 \theta \leq 45^{\circ}$ para WAXD, onde $2 \theta$ é o ângulo de espalhamento e $q=4 \pi \operatorname{sen}(\theta) / \lambda$, sendo $\lambda$ o comprimento de onda. Ambos detectores estão sincronizados e um tempo mínimo de coleta de 30 ou 60 segundos é necessário para obter uma estatística razoável em cada registro ("frame").

\section{Experimental}

\section{Preparação das amostras}

Os homopolímeros policaprolactona (PCL), $<\mathrm{M}_{\mathrm{w}}>=65.000$ $\mathrm{g} / \mathrm{mol}<\mathrm{M}_{\mathrm{w}}>/<\mathrm{M}_{\mathrm{n}}>=1,44$, e polietileno clorado ( $\mathrm{PECl}$ ) com fração em massa de cloro de $48 \%,<\mathrm{M}_{\mathrm{w}}>=43.520 \mathrm{~g} / \mathrm{mol}<\mathrm{M}_{\mathrm{w}}>/$ $<\mathrm{M}_{\mathrm{n}}>=1,7$ (determinado por GPC), foram comprados da Aldrich Co. As blendas PCL/PECl com as composições: 90/ $10,80 / 20,70 / 30$ e 60/40, foram obtidas a partir da mistura das soluções dos homopolímeros em tolueno nas proporções desejadas. Após a homogeneização o solvente foi removido por evaporação em estufa a vácuo a $35^{\circ} \mathrm{C}$ por 21 dias, obtendo-se filmes de cerca de $1 \mathrm{~mm}$ de espessura.

\section{Medidas de DSC}

As amostras foram seladas em um porta-amostra de alu- 
mínio de DSC para amostras sólidas, modificado como descrito anteriormente. Tipicamente a massa utilizada em cada análise variou entre 5 e $10 \mathrm{mg}$. Foram realizados experimentos dinâmicos e experimentos de cristalização isotérmica combinados com medidas simultâneas de SAXS e WAXD.

\section{Experimentos dinâmicos}

A PCL e as blendas contendo 80 e $60 \%$ em massa de PCL foram analisadas a $20^{\circ} \mathrm{C} / \mathrm{min}$ sob fluxo de nitrogênio. Os experimentos foram feitos seguindo o programa: i) aquecimento a partir da temperatura ambiente até $120^{\circ} \mathrm{C}$ e tratamento isotérmico nesta temperatura por 5 minutos; ii) resfriamento até $-120^{\circ} \mathrm{C}$ e estabilização nesta temperatura durante $5 \mathrm{mi}$ nutos; iii) aquecimento até $120^{\circ} \mathrm{C}$.

\section{Cristalização isotérmica e fusão}

Após a eliminação da historia térmica do material, as amostras foram resfriadas a uma taxa de $3^{\circ} \mathrm{C} / \mathrm{min}$ até atingir a temperatura de tratamento térmico, $T_{c}$. Então, foram mantidas nesta temperatura até não serem observadas mudanças nos difratogramas (intensidade dos picos cristalinos constante). As temperaturas de cristalização utilizadas foram: 39, 42, 45
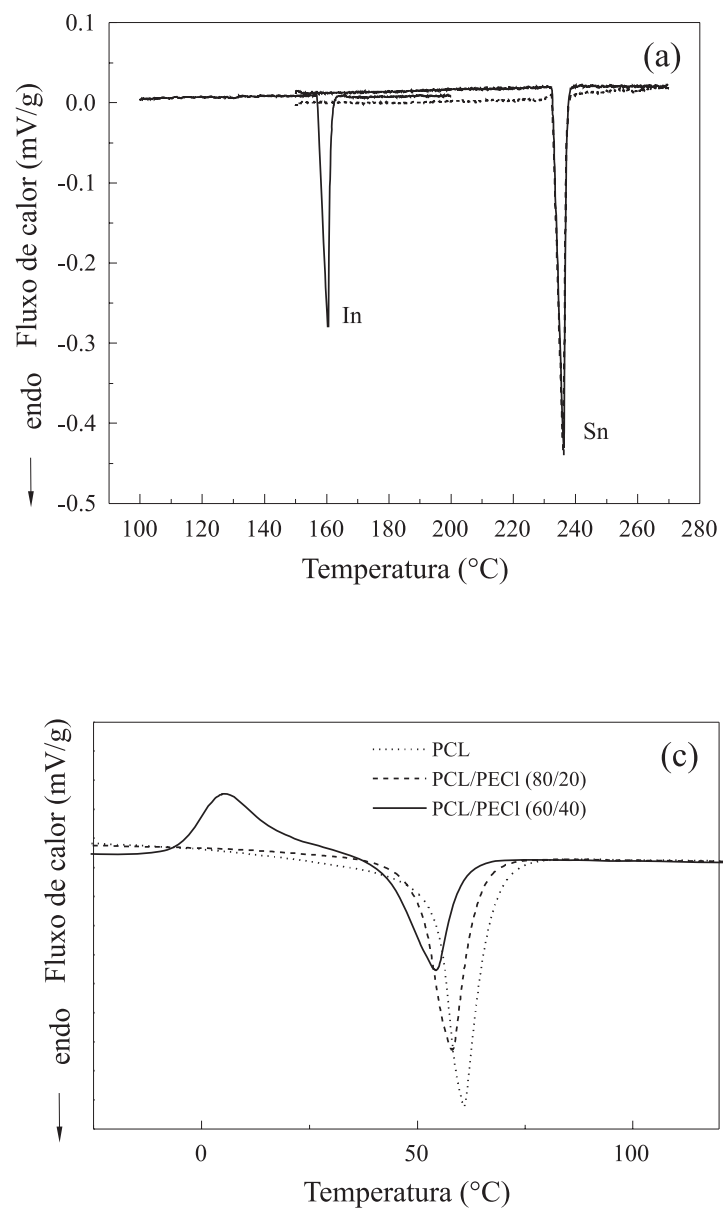

e $47^{\circ} \mathrm{C}$. O ciclo de fusão de cada amostra foi feito resfriando-se o material de $T_{c}$ até a temperatura ambiente $\left(25^{\circ} \mathrm{C}\right)$, mantendo-se nesta temperatura por 5 minutos e aquecendose a amostra a $10^{\circ} \mathrm{C} / \mathrm{min}$ até $120^{\circ} \mathrm{C}$.

\section{Medidas de SAXS/WAXD}

Os experimentos de espalhamento de raios $\mathrm{X}$ foram realizados com um comprimento de onda de 1,743 $\AA$. Cada curva de espalhamento foi acumulada durante 30 segundos e o registro total dos dados se estendeu durante todo o tratamento térmico programado (entre 30 minutos e 3 horas para as diferentes temperaturas e composições estudadas). As posições angulares foram calibradas com um padrão de parafina (n-tetracosano).

\section{Resultados e Discussão}

\section{Características específicas do DSC/THM 600}

Medidas realizadas com padrões índio e estanho permitiram observar alta repetitividade e a reprodutibilidade ${ }^{[6]}$ do equipamento de DSC, tanto em relação ao perfil da curva obtida como em relação aos valores de temperatura das tran-
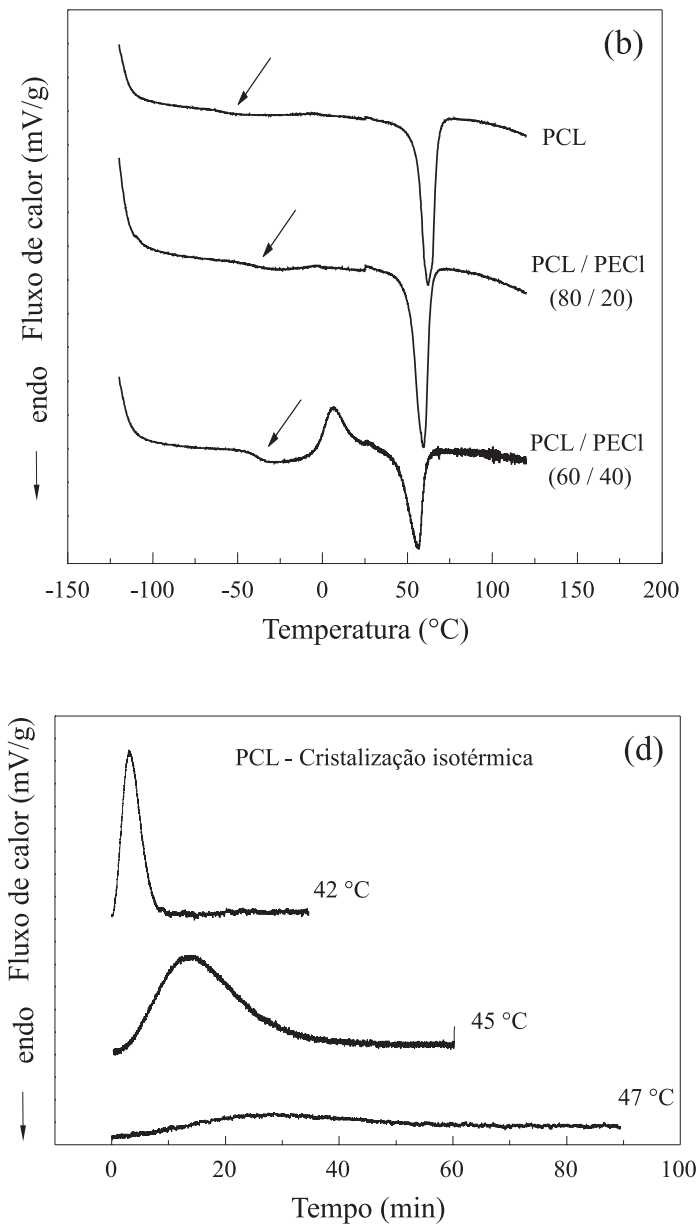

Figura 2. (a) Curvas de DSC obtidas em dois experimentos diferentes sob mesmas condições experimentais para os padrões índio (In) e estanho (Sn). As curvas foram corrigidas em relação à curva de referência e à massa da amostra; (b) curvas obtidas para PCL e para suas blendas contendo 20 e $40 \%$ em massa de $\mathrm{PECl}$ durante o aquecimento a partir de -120 a $120^{\circ} \mathrm{C}$ a $20^{\circ} \mathrm{C} / \mathrm{min}$ (flecha indica a região onde acontece à transição vítrea); (c) detalhe de b nas temperaturas de fusão dos materiais mostrando a diferencia de intensidade dos picos endotérmicos; (d) cristalização isotérmica da PCL a 42,45 e $47^{\circ} \mathrm{C}$. 
Tabela 1. Temperatura de transição vítrea $\left(\mathrm{T}_{\mathrm{g}}\right)$ e temperatura de fusão $\left(\mathrm{T}_{\mathrm{m}}\right)$ para PCL e as blendas PCL/PECl nas composições 80/20 e 60/40

\begin{tabular}{ccc}
\hline Amostra & $\mathbf{T}_{\mathbf{g}}\left({ }^{\circ} \mathbf{C}\right)$ & $\mathbf{T}_{\mathbf{m}}\left({ }^{\circ} \mathbf{C}\right)$ \\
\hline PCL & -60 & 62 \\
$80 / 20$ & -41 & 59 \\
$60 / 40$ & -40 & 56 \\
\hline
\end{tabular}

sições de primeira ordem (fusão). Na Figura 2a observa-se que as duas curvas corrigidas para cada padrão e obtidas em diferentes análises são muito similares. Em todas elas a inclinação da linha de base é constante indicando que a subtração pela referência é precisa.

Ainda com a finalidade de observar as características deste equipamento, foram realizados alguns testes com amostras poliméricas, sendo um deles conduzido a partir da temperatura subambiente para a verificação do funcionamento do sistema de resfriamento (Figura 2b); e o outro realizado em condição isotérmica para a verificação da estabilidade da temperatura do forno (Figura 2d). Estes ensaios foram realizados com a policaprolactona (PCL), um polímero semi-cristalino que apresenta alta cristalinidade e que garante um pico de fusão bem definido e intenso, e com blendas de PCL contendo 20 e $40 \%$ de PECl.

$\mathrm{Na}$ Figura 2b, encontram-se as curvas de DSC obtidas a partir da temperatura subambiente. As transições estão bem definidas e os valores determinados para a temperatura de transição vítrea e de fusão são comparáveis aos obtidos no equipamento DSC TA 2910 ${ }^{[7]}$. Para a blenda PCL/PECl 60/ 40 observa-se também um pico exotérmico entre a transição vítrea e a fusão da amostra, devido à recristalização durante o aquecimento. Os valores da temperatura de transição vítrea $\left(T_{g}\right)$ e a temperatura de fusão $\left(T_{m}\right)$ como função da composição da blenda é apresentada na Tabela 1. Observa-se o deslocamento da $T_{g}$ para temperaturas maiores com o acréscimo do teor de $\mathrm{PECl}$ nas blendas, como esperado para sistemas miscíveis. Além disso a temperatura de fusão se desloca para menores temperaturas ao mesmo tempo em que a intensidade do pico endotérmico é reduzida (Figura 2c). Os resultados dos experimentos isotérmicos são apresentados na Figura 2d, onde pode ser verificado que o forno mantém a temperatura uniforme durante o período de tempo do experimento, sendo possível a alteração de poucos graus $\left(2{ }^{\circ} \mathrm{C}\right)$ entre diferentes experimentos. A variação diferencial alcançada com este forno é pequena. Isto é importante em experimentos de cristalização isotérmica e nem sempre se consegue em equipamentos convencionais que possuem fornos com dimensões maiores.

\section{Medidas de SAXS/WAXD/DSC}

As análises simultâneas SAXS/WAXD/DSC foram realizadas em condições de cristalização isotérmica para a PCL e suas blendas.

O sistema PCL/PECl é miscível em toda a faixa de com-
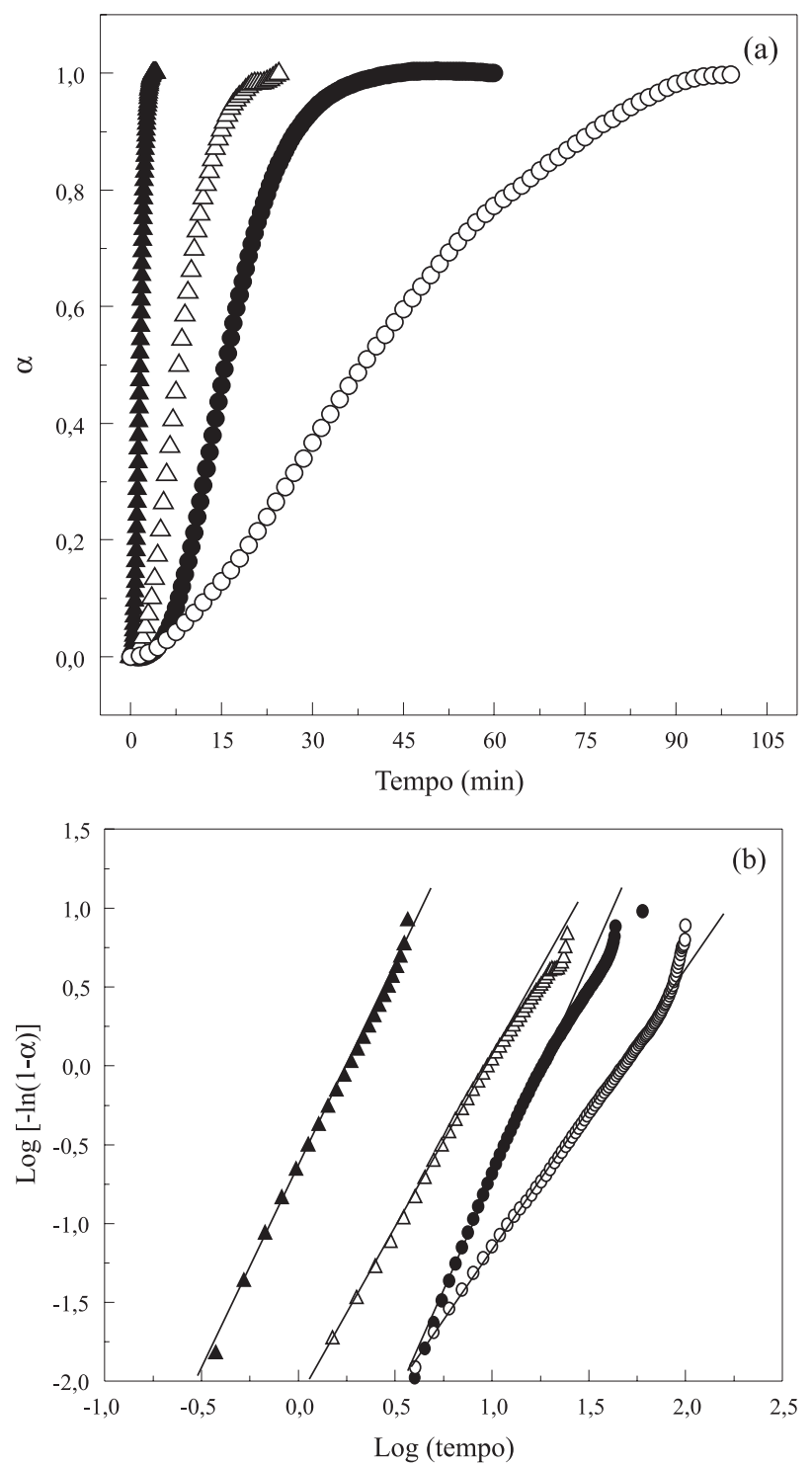

Figura 3. (a) Curvas do grau de conversão em função tempo obtidas em diferentes temperaturas de cristalização. (b) Ajustes obtidos a partir da equação de Avrami. PCL: $(\boldsymbol{\Delta}) \mathrm{T}_{\mathrm{c}}=39^{\circ} \mathrm{C}$ e $(\bullet) \mathrm{T}_{\mathrm{c}}=45^{\circ} \mathrm{C}$; blenda PCL/PECl $(80 /$ 20): $(\Delta) \mathrm{T}_{\mathrm{c}}=39^{\circ} \mathrm{C}$ e (o) $\mathrm{T}_{\mathrm{c}}=45^{\circ} \mathrm{C}$.

posição, apresentando uma única temperatura de transição vítrea, que ocorre em valores intermediários aos observados para os polímeros puros ${ }^{[7]}$.

Nas curvas de DSC é possível observar o efeito da adição do PECl na cinética de cristalização da PCL. Na Figura 3a são mostrados as curvas do grau de conversão, $\alpha$, em função do tempo de tratamento térmico para a PCL e a blenda PCL/ PECl 80/20. Observa-se ainda que o aumento da temperatura do tratamento isotérmico torna a cristalização mais lenta, tanto para a PCL pura como para a blenda PCL/PECl 80/20, como é esperado.

Em geral, os estágios iniciais da cristalização (cristalização primária) podem ser analisados utilizando-se a equação de Avrami ${ }^{[8]}$ :

$$
\log [-\ln (1-\alpha(t))]=\log K_{n}+n \log t
$$


Tabela 2. expoente de Avrami, $n$, como função da temperatura da isoterma e da composição da blenda. Temperatura de fusão do equilíbrio, $\mathrm{T}_{\mathrm{m}}{ }^{0}$ obtida a partir do gráfico de Hoffmann-Weeks.

\begin{tabular}{cccc}
\hline $\mathbf{T}_{\mathbf{c}}\left({ }^{\circ} \mathbf{C}\right) / \mathbf{n}$ & $\mathbf{P C L}$ & $\mathbf{8 0 / 2 0}$ & $\mathbf{7 0 / 3 0}$ \\
\hline 36 & & 2,2 & 2,6 \\
37 & & 2,4 & 2,6 \\
39 & 2,5 & 2,2 & 2,5 \\
42 & 2,5 & 1,8 & 1,8 \\
45 & 3,1 & 1,7 & 1,9 \\
47 & 2,6 & 1,6 & 1,7 \\
$\mathrm{~T}_{\mathrm{m}}{ }^{0}\left({ }^{\circ} \mathrm{C}\right)$ & 71 & 69 & 68 \\
\hline
\end{tabular}

onde $K_{n}$ é a constante de velocidade e $n$ é um parâmetro adimensional que depende do mecanismo de nucleação e da forma de crescimento dos cristais em 1, 2 ou 3 dimensões. A Figura 3 b exemplifica alguns dos ajustes obtidos.

Os valores de $n$ obtidos para PCL e algumas das composições utilizadas são mostrados na Tabela 2. Em todos os casos os valores de $n$ foram não inteiros, indicando que ocorrem paralelamente mecanismos de crescimento e/ou nucleação secundária superficial ${ }^{[9]}$. Para a PCL o valor fica em torno de 2,5 , independentemente da temperatura de cristalização, sendo coerente com outros resultados descritos na literatura ${ }^{[10]}$. Para a blenda em temperaturas de cristalização inferiores ou iguais a $39^{\circ} \mathrm{C}$ o valor de $n$ é semelhante ao da PCL pura, porém, para temperaturas de cristalização maiores que $39^{\circ} \mathrm{C}$ o valor de $n$ diminui indicando mudanças no mecanismo de nucleação e crescimento da PCL, o que provavelmente é causado pela presença das cadeias do $\mathrm{PECl}$.

Durante a fusão das amostras todas as curvas de DSC obtidas para a $\mathrm{PCl}$ e as blendas apresentaram apenas um pico endotérmico. Utilizando-se o método de Hoffmann-Weeks ${ }^{[11]}$ foi determinada a temperatura de fusão de equilíbrio $\left(T_{m}{ }^{o}\right)$ de cada blenda. Os valores para algumas composições são indicados na Tabela 2. Para a PCL pura o valor obtido é coerente com os valores descritos na literatura ${ }^{[9]}$. Nas blendas observou-se uma leve depressão do ponto de fusão com o aumento do teor de $\mathrm{PECl}$. Esse resultado é esperado considerando que o sistema é miscível, ainda que o parâmetro de interação polímero/polímero de Flory-Huggins seja próximo a zero ${ }^{[12]}$.

Durante o tratamento isotérmico da amostra no DSC foram obtidos registros sucessivos de SAXS/WAXD. Resultados típicos são apresentados na Figura 4 e 5 para a PCL tratada isotermicamente a $T_{c}=42{ }^{\circ} \mathrm{C}$ por 35 min. Na Figura 4a são mostrados os resultados de SAXS, onde observa-se que o pico característico associado à periodicidade lamelar surge bem definido no início da cristalização (terceiro "frame", $t=2$ min), enquanto que no mesmo instante de tempo o difratograma na medida de WAXD (Figura 4b) apresenta as reflexões principais da $\mathrm{PCL}$ associadas às distâncias interplanares $(d=4,12 \AA$ e $d=3,73 \AA)$. Durante o tempo de tratamento isotérmico é verificado o crescimento (aumento

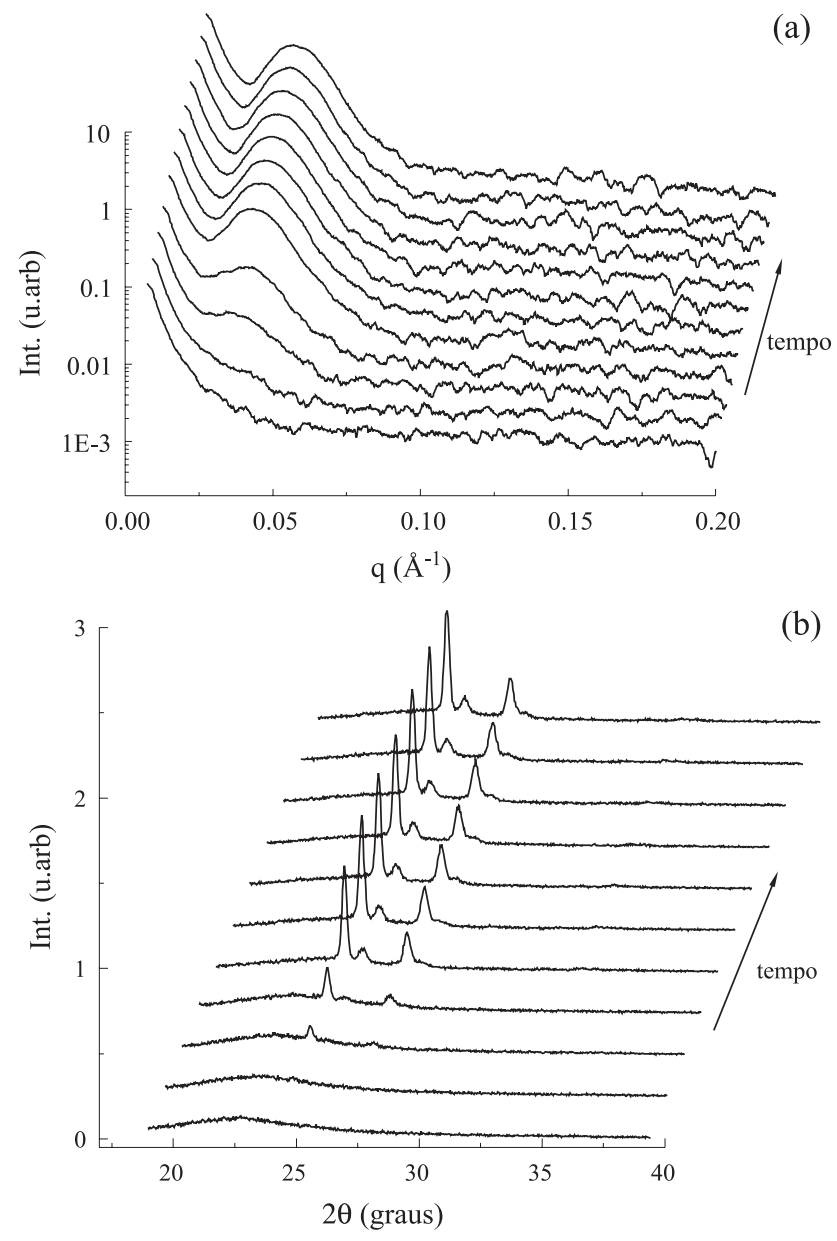

Figura 4. Evolução temporal das curvas de (a) SAXS e (b) WAXD durante a cristalização da $\mathrm{PCL}$ a $\mathrm{T}_{\mathrm{c}}=42{ }^{\circ} \mathrm{C}$ por $35 \mathrm{~min}$. $\mathrm{O}$ espaçamento em tempo entre as primeiras cinco curvas é de 1 min e de 5 min nas restantes.

da fração volumétrica da fase cristalina) e a estabilização das estruturas lamelares e cristalinas.

Quando inicia-se a fusão observa-se por SAXS (Figura 5a) um deslocamento do pico lamelar para valores menores de $q$ até desaparecer completamente em $t=49 \mathrm{~min}$. Esse instante de tempo corresponde à fusão completa da fase cristalina da PCL, fato que pode ser corroborado pelo desaparecimento dos picos cristalinos nas experiências de WAXD (neste instante de tempo apenas é observado um halo amorfo, ver Figura 5b).

A evolução dos parâmetros lamelares da PCL (período longo, $L$, espessura lamelar, $l_{c}$, e espessura interlamelar amorfa, $l_{a}$ ) durante cada ciclo de temperatura, pode ser analisada assumindo um modelo ideal bifásico em escala nanométrica para a morfologia do sistema observado, e fazendo o cálculo da função de correlação unidimensional dos espectros de $\mathrm{SAXS}^{[13]}$. O ciclo térmico programado e os valores obtidos por SAXS como função do tempo de tratamento térmico podem ser observados nas Figuras $6 \mathrm{a}$ e $6 \mathrm{~b}$. Na última figura também são representados os valores do período longo obtidos diretamente da posição do máximo no gráfico $I q^{2}$ contra $q$ pela equação $L_{B}=2 \pi / q_{m}$. Para fins comparativos é mostrada a curva de conversão em função do tempo do tratamento isotérmico (Figura 6c). 

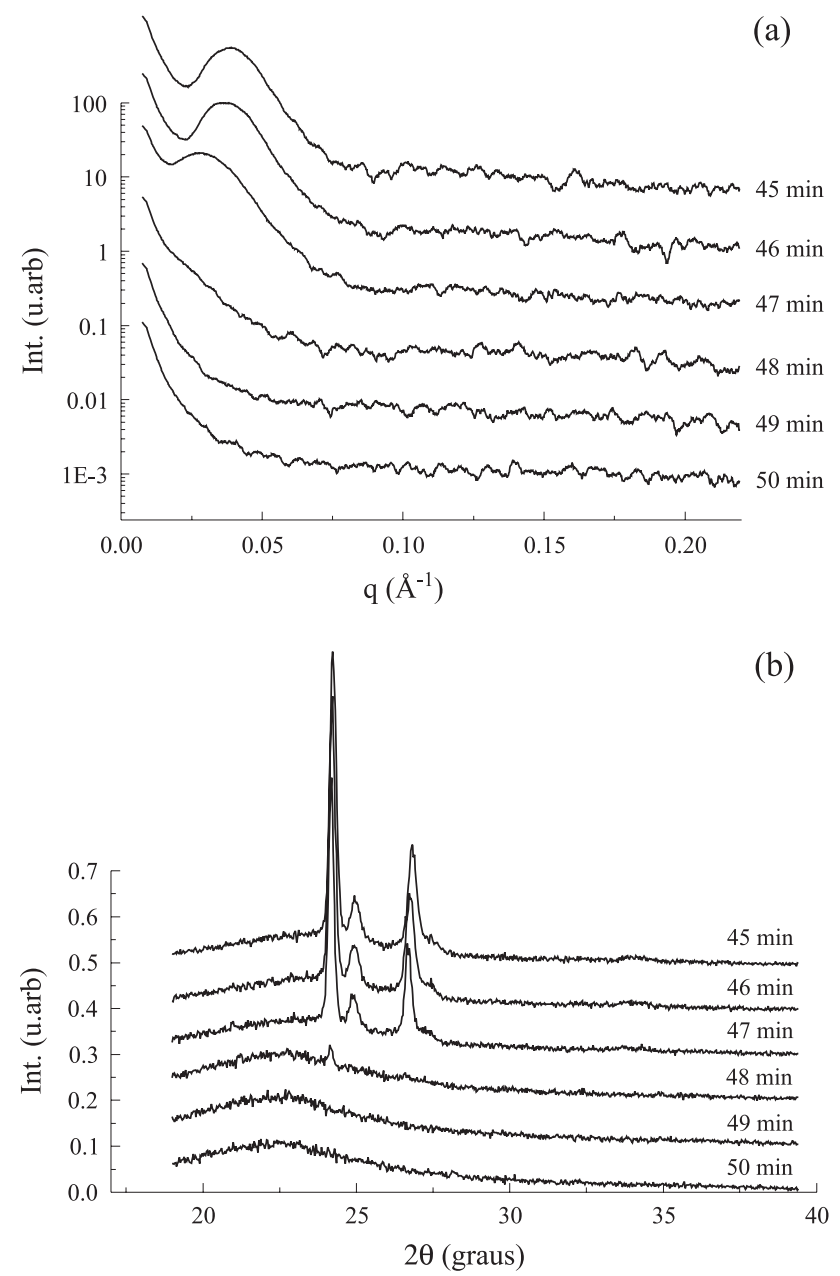

Figura 5. Fusão da PCL observada por (a) SAXS e (b) WAXD durante o aquecimento a $10{ }^{\circ} \mathrm{C} / \mathrm{min}$ a partir da temperatura ambiente até $120^{\circ} \mathrm{C}$.

No estágio inicial da cristalização (primeiros 5 minutos), observa-se claramente que o período longo e a espessura lamelar diminuem em função do tempo de tratamento térmico. Este fato é descrito na literatura para a $\mathrm{PCL}^{[14]}$ e para outros poliésteres ( $\mathrm{PET}^{[15]}$ e $\mathrm{PBT}^{[16]}$ ), sendo interpretado como a formação de lamelas secundárias, de menor espessura localizadas entre as lamelas primárias. Após este primeiro intervalo de tempo, no restante do tratamento isotérmico e dentro dos erros experimentais, os valores dos parâmetros lamelares permanecem constantes. O intervalo de tempo onde estes parâmetros começam a ser constantes é coincidente com a estabilização do máximo da curva de conversão. O resfriamento até $25{ }^{\circ} \mathrm{C}$ e o tratamento isotérmico por $5 \mathrm{~min}$ (intervalo de tempo entre os 35 e 45 min na Figura 6b) completa a cristalização do sistema possibilitando a formação de lamelas de espessura menor. Assim os parâmetros $L$ e $l_{c}$ diminuem. Finalmente, durante o ciclo de aquecimento (acima de $45 \mathrm{~min}$ ) e antes de fundir toda a fase cristalina, os valores médios $L$ e $l_{c}$ aumentam, devido à fusão preferencial das lamelas de espessura menor.

O efeito da composição na morfologia e na estrutura da blenda pode ser analisado no final do tratamento isotérmico, uma vez que o sistema atingiu a sua estabilização. Na Figura
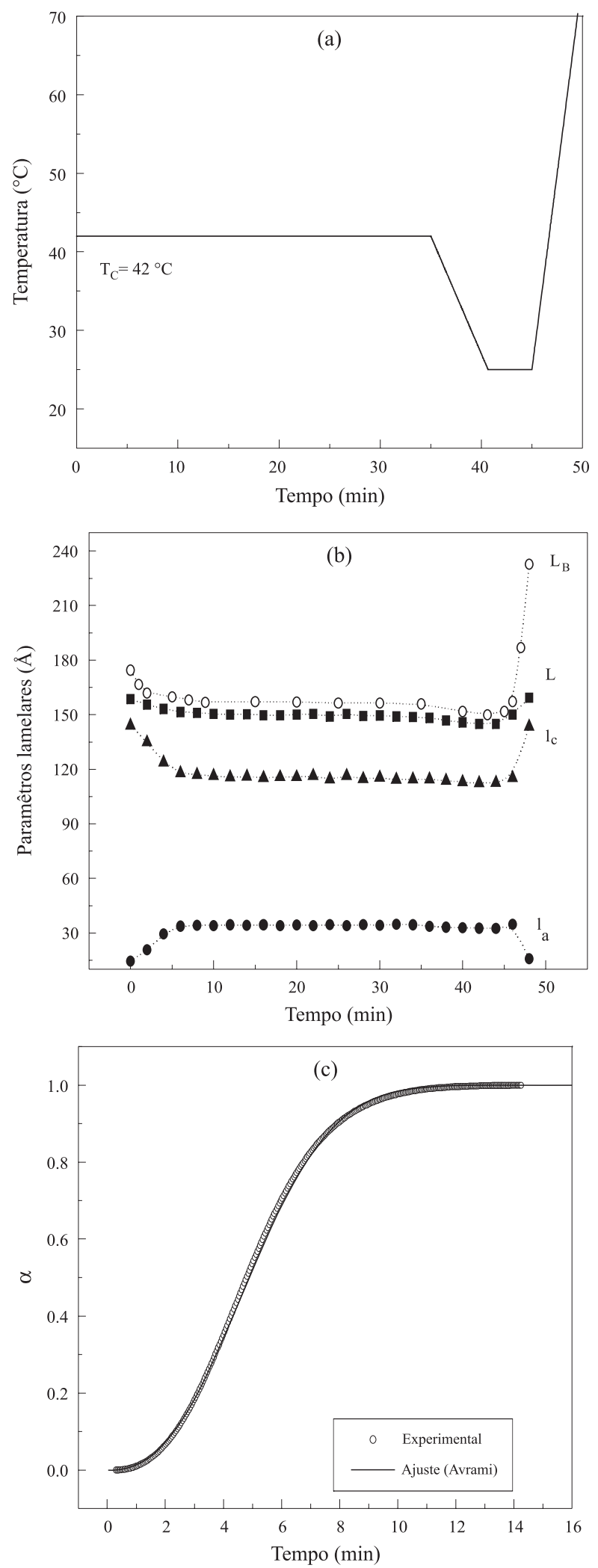

Figura 6. (a) Ciclo térmico programado. (b) Parâmetros lamelares em função da corrida de temperatura programada. $\mathrm{L}_{\mathrm{B}}$ : período longo obtido da posição do máximo no gráfico I q ${ }^{2}$ contra q; L: período longo, $1_{\mathrm{c}}$ : espessura lamelar, $1_{\mathrm{a}}$ : espessura interlamelar amorfa, obtidos através do cálculo da função de correlação unidimensional. (c): grau de conversão em função do tempo obtido pela curva de DSC realizada simultaneamente aos experimentos de SAXS e WAXD. 

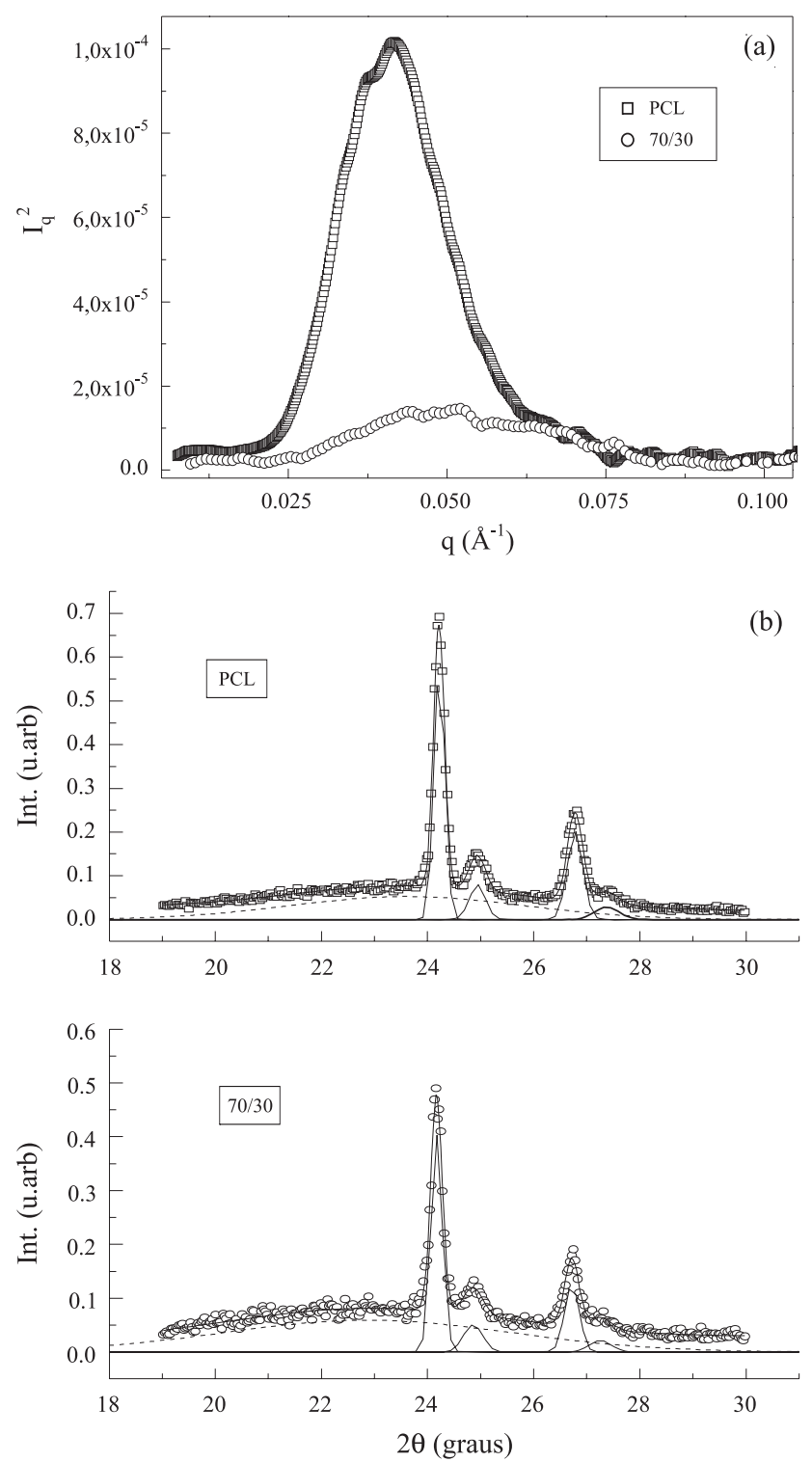

Figura 7. (a) Curvas de SAXS e (b) WAXD no final do tratamento térmico a $\mathrm{T}=42{ }^{\circ} \mathrm{C}$ em PCL na blenda 70/30. Em (b) pontos: dados experimentais, linhas: ajuste obtido (linha tracejada: halo amorfo, linha contínua: picos cristalinos)

7a-b são apresentadas as curvas de SAXS (na forma $I q^{2}$ contra $q$ ) e os difratogramas para PCL e para a blenda contendo $30 \%$ em massa de PECl tratadas a $\mathrm{T}_{\mathrm{c}}=42^{\circ} \mathrm{C}$. Por SAXS é evidente o deslocamento do pico de correlação para q maiores na blenda. Este fato revela uma redução do período longo que pode ser atribuída à redução da espessura lamelar da PCL e à localização preferencial da $\mathrm{PECl}$ entre os ramos lamelares (fibrilas) de PCL ${ }^{[17]}$. Por WAXD observa-se que as posições dos picos cristalinos (linhas contínuas) não mudam com a adição de $\mathrm{PECl}$, indicando, como era esperado, que a estrutura cristalina da PCL não é alterada pela presença do segundo polímero. Neste caso, o $\mathrm{PECl}$ apenas contribui para o halo amorfo (linha tracejada) que apresenta deslocamento do centro e aumento da intensidade relativa em relação aos picos cristalinos.

A boa resolução temporal obtida neste tipo de experi-
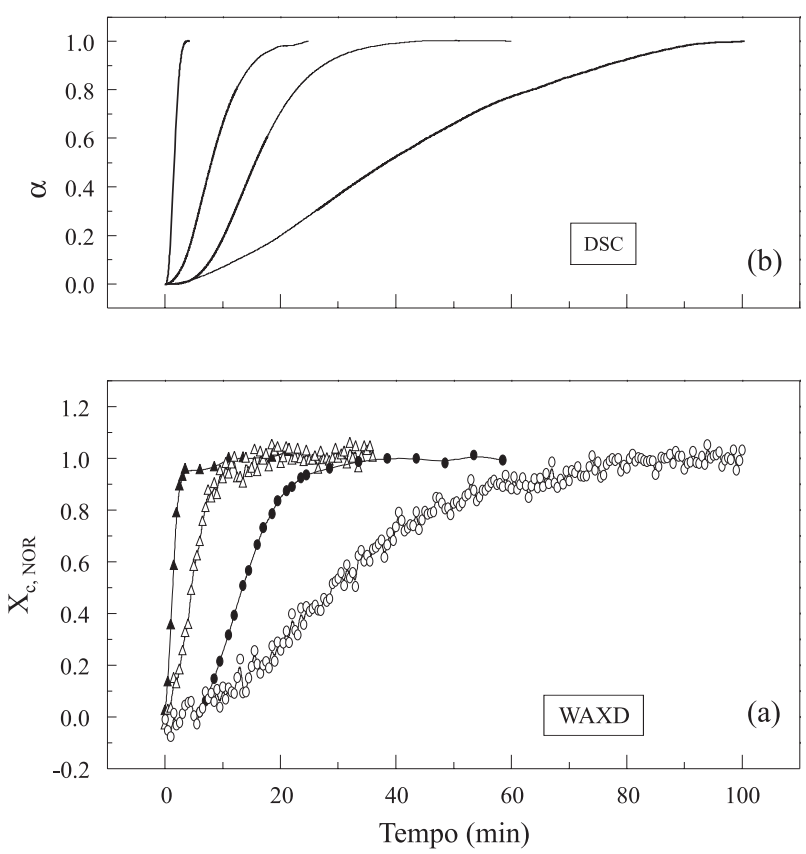

Figura 8. (a): grau de cristalinidade em relação à massa, normalizado pelo seu valor máximo $\left(X_{c, N O R}\right)$ em função do tempo de tratamento térmico para PCL: $(\Delta) \mathrm{T}_{\mathrm{c}}=39^{\circ} \mathrm{C} \mathrm{e}(\bullet) \mathrm{T}_{\mathrm{c}}=45^{\circ} \mathrm{C}$ e a blenda PCL/PECl $(80 / 20):(\Delta) \mathrm{T}_{\mathrm{c}}=39^{\circ} \mathrm{C}$ e (o) $\mathrm{T}_{\mathrm{c}}=45^{\circ}{ }^{\circ} \mathrm{C}$. (b): grau de conversão em função do tempo obtido simultaneamente das medidas de DSC.

mentos permite calcular com precisão o grau de cristalinidade da blenda em função do tempo transcorrido da cinética, a partir do quociente da área total relativa aos picos cristalinos em relação à área total difratada (picos cristalinos e halo amorfo). A evolução deste parâmetro normalizado pelo seu valor máximo $\left(X_{c, N O R}\right)$ é mostrado na Figura 8a para a PCL pura e a blenda $80 / 20$ nas temperaturas de cristalização de $39^{\circ} \mathrm{C}$ e $45^{\circ} \mathrm{C}$. Estes resultados são qualitativamente comparáveis aos obtidos pelo DSC (Figura 8 b) mostrando tanto o efeito retardador da temperatura do tratamento isotérmico como do polímero amorfo na velocidade de cristalização da PCL.

\section{Conclusões}

A utilização das técnicas de SAXS e WAXD simultaneamente com a técnica calorimétrica, foi testada e caracterizada em experimentos em tempo real de amostras poliméricas em uma faixa de temperaturas ou em experimentos isotérmicos, na linha de SAXS do LNLS. A célula de DSC, modificada para permitir a transmissão dos raios $\mathrm{X}$, apresentou desempenho adequado e comparável aos equipamentos convencionais de DSC em relação à repetitividade e reprodutibilidade tanto em análises de padrões como em exames de amostras poliméricas. O equipamento pode ser utilizado em condições dinâmicas de aquecimento ou em condições isotérmicas com alta confiabilidade. Transições de primeira ordem, como a fusão e a cristalização, e transições de pseudosegunda ordem, como a transição vítrea, puderam ser determinadas com precisão. A potencialidade desse arranjo experimental foi exemplificada na análise da cristalização 
isotérmica da PCL e da blenda miscível semi-cristalina PCL/ $\mathrm{PECl}$. Os experimentos de DSC e WAXD permitem visualizar a influência das cadeias de $\mathrm{PECl}$ na cinética de cristalização da PCL, observando-se uma redução na velocidade de cristalização com o aumento no teor da $\mathrm{PECl}$. A análise utilizando a equação de Avrami mostrou mudanças dos mecanismos de nucleação e crescimento dependentes da temperatura e da composição da blenda. O estudo combinado de SAXS/WAXD (em tempo real) permitiu que se observe a simultaneidade dos eventos de formação dos cristais da PCL e da periodicidade da estrutura lamelar. As medidas de SAXS em tempo real e sua comparação com as varreduras de DSC mostraram que durante a cristalização primária o período longo e a espessura lamelar diminuíram com o tempo de tratamento térmico enquanto que na fase final da cristalização, para os tempos considerados, não se observaram efeitos de "annealing" (a espessura lamelar é constante com o tratamento térmico). Finalmente cabe destacar que a utilização simultânea das técnicas SAXS/WAXD/ DSC, permite não apenas os estudos de cristalização, mas também análises de formação de colóides e géis, acompanhamento de transições ordem-desordem e de processos de transesterificação entre outros.

\section{Agradecimentos}

Pesquisa desenvolvida baixo o projeto D11A-SAXS número 1709, LNLS-CNPq. Gostaríamos de agradecer ao Prof. Dr. Wim Bras, ESRF, pela doação do estágio DSC/THM 600 e ao Dr. G. Kellermann pela ajuda no desenho do arranjo experimental no LNLS. Agradecemos também ao Dr. G. Mantovani e a Profa. Dra M.I. Felisberti por produtivas discussões na estratégia de caracterização do DSC e pelas curvas de DSC obtidas no TA 2910 no Instituto de Química da Universidade Estadual de Campinas.

\section{Referências Bibliográficas}

1. Bras, W.; Derbyshire, G. E.; Devine, A.; Clark, S. M.; Komanschek, B. E. \& Ryan, A. J.- J. Appl. Cryst 28, p.26 (1995).

2. Ryan, A. J. - J. Thermal Anal. 40, p.887 (1993).
3. Terrill, N. J.; Fairclough, P. A.;Towns-Andrews, E.; Komanschek, B. U.; Ypung, R. J. \& Ryan, A. J. Polymer 39, p.2381 (1998).

4. Russell, T. P \& Koberstein, J. T.- J. Polym. Sci. Polym. Phys. Ed. 23, p.1109 (1985).

5. Kellermann, G.; Vicentin, F.; Tamura, E.; Rocha, M.; Tolentino, H.; Barbosa, A.; Craievich, A. F. \& Torriani, I. - J. Appl. Cryst. 30, p.880 (1997).

6. Kateman, G. \& Pijpers, F. W. , in "Quality Control in Analytical Chemistry", vol 60 p.9, Kateman, G.\& Pijpers, F.W. (ed.), John Wiley \& Sons New York (1981).

7. Plivelic, T. S.; Cassu, S. N.; Torriani, I. L. \& Gonçalves, M. C. -Activity report LNLS 2001 p.166 (2002).

8. Avrami, M. J. - J. Chem. Phys 7, p.1103 (1939).

9. Guo, Q. \& Groeninckx, G. - Polymer 42, p.8647 (2001).

10. Nojima, S.; Tsutsui, H.; Urushihara, M.; Kosaka, W.; Kato, N. \& Ashida, T. -Polym J 18, p.451 (1986).

11. Hoffmann, J. D. \& Weeks, J. J. - J.Res.Natl.Bur.Stand., Part A 66, p.13 (1962).

12. Bélorgey, G. \& Prud'Homme, R.E. -J. Polym. Sci. Polym. Phys. Ed. 20, p.191 (1982).

13. Strobl, G. R. \& Schneider, M. J. - J. Polym. Sci. Polym. Phys. Ed. 18, p.1343 (1980).

14. Nojima, S.; Kato, K.; Ono, M. \& Ashida T.-Macromolecules 25, p.1922 (1992).

15. Jonas, A. M.; Russell, T. P. \& Yoon, D. Y. - Colloid. Polym. Sci. 272, p.1344 (1994).

16. Hsiao, B. S.; Wang, Z. G.; Yeh, F. J.; Gao, Y. \& Sheth, K.Polymer 40, p.3515 (1999).

17. Plivelic, T. S.; Cassu, S. N.; Torriani, I. L. \& Gonçalves, M. C. -Anais do $7^{\circ}$ Congresso Brasileiro de Polímeros, Belo Horizonte-MG p.565 (2003).

Enviado: 10/09/04

Reenviado: 04/03/05

Aprovado: 15/03/05 